Honam Mathematical J. 35 (2013), No. 1, pp. 093-100

http://dx.doi.org/10.5831/HMJ.2013.35.1.93

\title{
STABILITY OF A QUADRATIC-ADDITIVE TYPE FUNCTIONAL EQUATION IN NON-ARCHIMEDEAN NORMED SPACES
}

\author{
Chang-Ju Lee And Yang-Hi Lee
}

\begin{abstract}
In this paper, we investigate the stability for the functional equation$$
2 f(x+y)+f(x-y)+f(y-x)-f(2 x)-f(2 y)=0
$$

in non-Archimedean normed spaces.
\end{abstract}

\section{Introduction}

We introduce some terminologies and notations used in the theory of non-Archimedean spaces (see [3]).

Definition 1.1. A field $\mathbb{K}$ equipped with a function (valuation) $|\cdot|$ from $\mathbb{K}$ into $[0, \infty)$ is called a non-Archimedean field if the function $|\cdot|: \mathbb{K} \rightarrow[0, \infty)$ satisfies the following conditions:

(i) $|r|=0$ if and only if $r=0$;

(ii) $|r s|=|r||s|$;

(iii) $|r+s| \leq \max \{|r|,|s|\}$ for all $r, s \in \mathbb{K}$.

Clearly, $|1|=|-1|$ and $|n| \leq 1$ for all $n \in \mathbb{N}$.

Definition 1.2. Let $X$ be a vector space over a scalar field $\mathbb{K}$ with a non-Archimedean nontrivial valuation $|\cdot|$. A function $\|\cdot\|: X \rightarrow \mathbb{R}$ is a non-Archimedean norm if it satisfies the following conditions:

(i) $\|x\|=0$ if and only if $x=0$;

(ii) $\|r x\|=|r|\|x\|(r \in \mathbb{K}, x \in X)$;

Received February 7, 2013. Accepted March 11, 2013.

2010 Mathematics Subject Classification. Primary 46S40; Secondary $39 B 52$.

Key words and phrases. non-Archimedean normed spaces, quadratic-additive type functional equation. (2012).

This work was supported by Gongju National University of Education Grant 
(iii) the strong triangle inequality, namely,

$$
\|x+y\| \leq \max \{\|x\|,\|y\|\}
$$

for all $x, y \in X$. In this case, the pair $(X,\|\cdot\|)$ is called a nonArchimedean space. Due to the fact that

$$
\left\|x_{n}-x_{m}\right\| \leq \max \left\{\left\|x_{j+1}-x_{j}\right\|: m \leq j \leq n-1\right\} \quad(n>m)
$$

a sequence $\left\{x_{n}\right\}$ is Cauchy if and only if $\left\{x_{n+1}-x_{n}\right\}$ converges to zero in a non-Archimedean space. By a complete non-Archimedean we mean one in which every Cauchy sequence is convergent.

The stability problem of the functional equation, was formulated by S. M. Ulam [5] in 1940. In the next year, D. H. Hyers [2] gave a partial solution of Ulam's problem for the case of an approximate additive mapping. Subsequently, Hyers' result was generalized by T. Aoki [1] for an additive mapping and by Th. M. Rassias [4] for a linear mapping with unbounded Cauchy differences.

Recently M. S. Moslehian and Th. M. Rassias [3] discussed the HyersUlam stability of the Cauchy functional equation

$$
f(x+y)=f(x)+f(y)
$$

and the quadratic functional equation

$$
f(x+y)+f(x-y)-2 f(x)-2 f(y)=0
$$

in non-Archimedean normed spaces.

Now we consider a quadratic-additive type functional equation

$$
2 f(x+y)+f(x-y)+f(y-x)-f(2 x)-f(2 y)=0
$$

which solution is called a quadratic-additive mapping. We remark that the general solution of the equation (1.3) is of the form $f(x)=Q(x)+$ $A(x)$, where $Q$ is a quadratic mapping and $A$ is an additive mapping.

In this paper, we get a general stability result of the quadraticadditive type functional equation (1.3) in non-Archimedean normed spaces.

\section{Stability of a quadratic-additive type functional equation}

Throughout this section, we assume that $X$ is a real linear space and $Y$ is a complete non-Archimedean space with $|2|<1$.

For a given mapping $f: X \rightarrow Y$, we use the abbreviation

$$
D f(x, y):=2 f(x+y)+f(x-y)+f(y-x)-f(2 x)-f(2 y)
$$


for all $x, y \in X$. Now, we will prove the stability of the quadraticadditive type functional equation (1.3).

Theorem 2.1. Let $\varphi: X^{2} \rightarrow[0, \infty)$ be a function such that

$$
\lim _{n \rightarrow \infty} \frac{\varphi\left(2^{n} x, 2^{n} y\right)}{|4|^{n}}=0, \quad(x, y \in X)
$$

and let for each $x \in X$ the limit

$$
\lim _{n \rightarrow \infty} \max \left\{\frac{\varphi\left(2^{j} x, 0\right)}{|2| \cdot|4|^{j+1}}, \frac{\varphi\left(-2^{j} x, 0\right)}{|2| \cdot|4|^{j+1}}: 0 \leq j<n\right\}
$$

denoted by $\tilde{\varphi}(x)$, exists. Suppose that $f: X \rightarrow Y$ is a mapping satisfying

$$
\|D f(x, y)\| \leq \varphi(x, y), \quad(x, y \in X) .
$$

Then there exists a unique quadratic-additive mapping $T: X \rightarrow Y$ such that

$$
\|f(x)-T(x)\| \leq \tilde{\varphi}(x), \quad(x \in X) .
$$

In particular, $T$ is given by

$$
T(x)=\lim _{n \rightarrow \infty} \frac{f\left(2^{n} x\right)+f\left(-2^{n} x\right)}{2 \cdot 4^{n}}+\frac{f\left(2^{n} x\right)-f\left(-2^{n} x\right)}{2^{n+1}}
$$

for all $x \in X$.

Proof. It follows from $|2|<1$ and (2.1) that

$$
\varphi(0,0) \leq \lim _{n \rightarrow \infty} \frac{\varphi(0,0)}{|4|^{n}}=0 .
$$

So we have

$$
\|2 f(0)\|=\|D f(0,0)\| \leq \varphi(0,0)=0,
$$

i.e., $f(0)=0$. Let $J_{n} f: X \rightarrow Y$ be a function defined by

$$
J_{n} f(x)=\frac{f\left(2^{n} x\right)+f\left(-2^{n} x\right)}{2 \cdot 4^{n}}+\frac{f\left(2^{n} x\right)-f\left(-2^{n} x\right)}{2^{n+1}}
$$

for all $x \in X$ and $n \in \mathbb{N}$. Notice that $J_{0} f(x)=f(x)$ and

$$
\begin{aligned}
& \left\|J_{j} f(x)-J_{j+1} f(x)\right\| \\
= & \left\|\frac{D f\left(2^{j} x, 0\right)}{2 \cdot 4^{j+1}}+\frac{D f\left(-2^{j} x, 0\right)}{2 \cdot 4^{j+1}}+\frac{D f\left(2^{j} x, 0\right)}{2^{j+2}}-\frac{D f\left(-2^{j} x, 0\right)}{2^{j+2}}\right\| \\
\leq & \max \left\{\frac{\left\|D f\left(2^{j} x, 0\right)\right\|}{|2| \cdot|4|^{j+1}}, \frac{\left\|D f\left(-2^{j} x, 0\right)\right\|}{|2| \cdot|4|^{j+1}}, \frac{\left\|D f\left(2^{j} x, 0\right)\right\|}{|2|^{j+2}}, \frac{\left\|D f\left(-2^{j} x, 0\right)\right\|}{|2|^{j+2}}\right\} \\
(2.5) \leq & \max \left\{\frac{\varphi\left(2^{j} x, 0\right)}{|2| \cdot|4|^{j+1}}, \frac{\varphi\left(-2^{j} x, 0\right)}{|2| \cdot|4|^{j+1}}\right\}
\end{aligned}
$$


for all $x \in X$ and $j \geq 0$. It follows from (2.1) and (2.5) that the sequence $\left\{J_{n} f(x)\right\}$ is Cauchy. Since $Y$ is complete, we conclude that $\left\{J_{n} f(x)\right\}$ is convergent. Set

$$
T(x):=\lim _{n \rightarrow \infty} J_{n} f(x) .
$$

Using induction one can show that

$$
\left\|J_{n} f(x)-f(x)\right\| \leq \max \left\{\frac{\varphi\left(2^{j} x, 0\right)}{|2|^{2 j+3}}, \frac{\varphi\left(-2^{j} x, 0\right)}{|2|^{2 j+3}}: 0 \leq j<n\right\}
$$

for all $x \in X$ and $n \in \mathbb{N}$. By taking $n$ to approach infinity in (2.6) and using (2.2), one obtains (2.4). Replacing $x$ and $y$ by $2^{n} x$ and $2^{n} y$, respectively, in $(2.3)$, we get

$$
\begin{array}{r}
\left\|D J_{n} f(x, y)\right\|=\| \frac{D f\left(2^{n} x, 2^{n} y\right)-D f\left(-2^{n} x,-2^{n} y\right)}{2^{n+1}} \\
+\frac{D f\left(2^{n} x, 2^{n} y\right)+D f\left(-2^{n} x,-2^{n} y\right)}{2^{2 n+3}} \| \\
\leq \max \left\{\frac{\varphi\left(2^{n} x, 2^{n} y\right)}{|2|^{n+1}}, \frac{\varphi\left(-2^{n} x,-2^{n} y\right)}{|2|^{n+1}},\right. \\
\left.\frac{\varphi\left(2^{n} x, 2^{n} y\right)}{|2| \cdot|4|^{n}}, \frac{\varphi\left(-2^{n} x,-2^{n} y\right)}{|2| \cdot|4|^{n}}\right\}
\end{array}
$$

for all $x, y \in X$ and $n \in \mathbb{N}$. Taking the limit as $n \rightarrow \infty$ and using (2.1), we get $D T(x, y)=0$. Thus, the mapping $T$ is a quadratic-additive mapping satisfying (2.4). Now, to prove that the quadratic-additive mapping $T$ is unique. If $T^{\prime}$ is another quadratic-additive mapping satisfying (2.4), then

$$
\begin{aligned}
& T^{\prime}(x)-J_{k} T^{\prime}(x) \\
= & \sum_{j=0}^{k-1}\left(\frac{\left(1+2^{j+1}\right) D T^{\prime}\left(2^{j} x, 0\right)}{2 \cdot 4^{j+1}}+\frac{\left(1-2^{j+1}\right) D T^{\prime}\left(-2^{j} x, 0\right)}{2 \cdot 4^{j+1}}\right) \\
= & 0
\end{aligned}
$$

for any $k \in \mathbb{N}$ and so 


$$
\begin{aligned}
& \left\|T(x)-T^{\prime}(x)\right\| \\
= & \lim _{k \rightarrow \infty}\left\|J_{k} T(x)-J_{k} T^{\prime}(x)\right\| \\
\leq & \lim _{k \rightarrow \infty} \max \left\{\left\|J_{k} T(x)-J_{k} f(x)\right\|,\left\|J_{k} f(x)-J_{k} T^{\prime}(x)\right\|\right\} \\
\leq & \lim _{k \rightarrow \infty}|2|^{-2 k-1} \max \left\{\left\|T\left(2^{k} x\right)-f\left(2^{k} x\right)\right\|,\left\|T\left(-2^{k} x\right)-f\left(-2^{k} x\right)\right\|,\right. \\
& \left.\left\|f\left(2^{k} x\right)-T^{\prime}\left(2^{k} x\right)\right\|,\left\|f\left(-2^{k} x\right)-T^{\prime}\left(-2^{k} x\right)\right\|\right\} \\
\leq & \lim _{k \rightarrow \infty} \lim _{n \rightarrow \infty} \max \left\{\frac{\varphi\left(2^{j} x, 0\right)}{|4|^{j+2}}, \frac{\varphi\left(-2^{j} x, 0\right)}{|4|^{j+2}}: k \leq j<n+k\right\} \\
= & 0 \quad(x \in X) .
\end{aligned}
$$

Therefore $T=T^{\prime}$. This completes the proof of the uniqueness of $T$.

Corollary 2.2. Let $X$ and $Y$ be non-Archimedean normed spaces over $\mathbb{K}$ with $|2|<1$. If $Y$ is complete and for some $2<r, f: X \rightarrow Y$ satisfies the condition

$$
\|D f(x, y)\| \leq \theta\left(\|x\|^{r}+\|y\|^{r}\right)
$$

for all $x, y \in X$. Then there exists a unique quadratic-additive mapping $T: X \rightarrow Y$ such that

$$
\|f(x)-T(x)\| \leq|2|^{-3} \theta\|x\|^{r} .
$$

Proof. Let $\varphi(x, y)=\theta\left(\|x\|^{r}+\|y\|^{r}\right)$. Since $|2|<1$ and $r-2>0$,

$$
\lim _{n \rightarrow \infty}|4|^{-n} \varphi\left(2^{n} x, 2^{n} y\right)=\lim _{n \rightarrow \infty}|2|^{n(r-2)} \varphi(x, y)=0
$$

for all $x, y \in X$. Therefore $f$ and $\varphi$ satisfy the conditions of Theorem 2.1. It is easy to see that $\tilde{\varphi}(x)=|2|^{-3} \theta\|x\|^{r}$. By Theorem 2.1 there is a unique quadratic-additive mapping $T: X \rightarrow Y$ satisfying (2.7).

Theorem 2.3. Let $\varphi: X^{2} \rightarrow[0, \infty)$ be a function such that

$$
\lim _{n \rightarrow \infty}|2|^{n} \varphi\left(2^{-n} x, 2^{-n} y\right)=0, \quad(x, y \in X)
$$

and let for each $x \in X$ the limit

(2.9) $\lim _{n \rightarrow \infty} \max \left\{|2|^{j-1} \varphi\left(\frac{x}{2^{j+1}}, 0\right),|2|^{j-1} \varphi\left(\frac{-x}{2^{j+1}}, 0\right): 0 \leq j<n\right\}$.

denoted by $\tilde{\varphi}(x)$, exists. Suppose that $f: X \rightarrow Y$ is a mapping satisfying

$$
\|D f(x, y)\| \leq \varphi(x, y), \quad(x, y \in X)
$$


with $f(0)=0$. Then there exists a unique quadratic-additive mapping $T: X \rightarrow Y$ such that

$$
\|f(x)-T(x)\| \leq \tilde{\varphi}(x), \quad(x \in X) .
$$

In particular, $T$ is given by

$T(x)=\lim _{n \rightarrow \infty}\left(2^{2 n-1}\left(f\left(\frac{x}{2^{n}}\right)+f\left(\frac{-x}{2^{n}}\right)\right)+2^{n-1}\left(f\left(\frac{x}{2^{n}}\right)-f\left(\frac{-x}{2^{n}}\right)\right)\right)$

for all $x \in X$.

Proof. Let $J_{n} f: X \rightarrow Y$ be a function defined by

$$
J_{n} f(x)=2^{2 n-1}\left(f\left(\frac{x}{2^{n}}\right)+f\left(\frac{-x}{2^{n}}\right)\right)+2^{n-1}\left(f\left(\frac{x}{2^{n}}\right)-f\left(\frac{-x}{2^{n}}\right)\right)
$$

for all $x \in X$ and $n \in \mathbb{N}$. Notice that $J_{0} f(x)=f(x)$ and

$$
\begin{aligned}
& \left\|J_{j} f(x)-J_{j+1} f(x)\right\| \\
= & \left\|-\frac{4^{j}+2^{j}}{2} D f\left(\frac{x}{2^{j+1}}, 0\right)-\frac{4^{j}-2^{j}}{2} D f\left(\frac{-x}{2^{j+1}}, 0\right)\right\| \\
\leq & \max \left\{|2|^{j-1} \varphi\left(\frac{x}{2^{j+1}}, 0\right),|2|^{j-1} \varphi\left(\frac{-x}{2^{j+1}}, 0\right)\right\}
\end{aligned}
$$

for all $x \in X$ and $j \geq 0$. It follows from (2.8) and (2.12) that the sequence $\left\{J_{n} f(x)\right\}$ is Cauchy. Since $Y$ is complete, we conclude that $\left\{J_{n} f(x)\right\}$ is convergent. Set

$$
T(x):=\lim _{n \rightarrow \infty} J_{n} f(x) .
$$

Using induction one can show that

$$
\begin{aligned}
\left\|J_{n} f(x)-f(x)\right\| \leq \max \left\{|2|^{j-1} \varphi\left(\frac{x}{2^{j+1}}, 0\right),\right. \\
\left.|2|^{j-1} \varphi\left(\frac{-x}{2^{j+1}}, 0\right): 0 \leq j<n\right\}
\end{aligned}
$$

for all $x \in X$ and $n \in \mathbb{N}$. By taking $n$ to approach infinity in (2.13) and using (2.9), one obtains (2.11). Replacing $x$ and $y$ by $2^{-n} x$ and $2^{-n} y$, respectively, in (2.10), we get

$$
\begin{aligned}
\left\|D J_{n} f(x, y)\right\|= & \| 2^{n-1} D f\left(\frac{x}{2^{n}}, \frac{y}{2^{n}}\right)-2^{n-1} D f\left(\frac{-x}{2^{n}}, \frac{-y}{2^{n}}\right) \\
& +2^{2 n-1} D f\left(\frac{x}{2^{n}}, \frac{y}{2^{n}}\right)+2^{2 n-1} D f\left(\frac{-x}{2^{n}}, \frac{-y}{2^{n}}\right) \| \\
\leq & \max \left\{|2|^{n-1} \varphi\left(\frac{x}{2^{n}}, \frac{y}{2^{n}}\right),|2|^{n-1} \varphi\left(\frac{-x}{2^{n}}, \frac{-y}{2^{n}}\right)\right\}
\end{aligned}
$$


for all $x, y \in X$ and $n \in \mathbb{N}$. Taking the limit as $n \rightarrow \infty$ and using (2.8), we get $D T(x, y)=0$. Thus, the mapping $T$ is a quadratic-additive mapping satisfying (2.11).

If $T^{\prime}$ is another quadratic-additive mapping satisfying (2.11), then

$$
T^{\prime}(x)-J_{k} T^{\prime}(x)=\sum_{j=0}^{k-1}\left(-\frac{4^{j}+2^{j}}{2} D f\left(\frac{x}{2^{j+1}}, 0\right)-\frac{4^{j}-2^{j}}{2} D f\left(\frac{-x}{2^{j+1}}, 0\right)\right)=0
$$

for any $k \in \mathbb{N}$ and so

$$
\begin{aligned}
& \left\|T(x)-T^{\prime}(x)\right\| \\
= & \lim _{k \rightarrow \infty}\left\|J_{k} T(x)-J_{k} T^{\prime}(x)\right\| \\
\leq & \lim _{k \rightarrow \infty} \max \left\{\left\|J_{k} T(x)-J_{k} f(x)\right\|,\left\|J_{k} f(x)-J_{k} T^{\prime}(x)\right\|\right\} \\
\leq & \lim _{k \rightarrow \infty}|2|^{k-1} \max \left\{\left\|T\left(\frac{x}{2^{k}}\right)-f\left(\frac{x}{2^{k}}\right)\right\|,\left\|T\left(-\frac{x}{2^{k}}\right)-f\left(-\frac{x}{2^{k}}\right)\right\|,\right. \\
\leq & \left.\lim _{k \rightarrow \infty}|2|^{k-1} \tilde{\varphi}\left(\frac{x}{2^{k}}\right)-T^{\prime}\left(\frac{x}{2^{k}}\right)\|,\| f\left(-\frac{x}{2^{k}}\right)-T^{\prime}\left(-\frac{x}{2^{k}}\right) \|\right\} \\
= & 0 \quad(x \in X) .
\end{aligned}
$$

Therefore $T=T^{\prime}$. This completes the proof of the uniqueness of $T$.

Corollary 2.4. Let $X$ and $Y$ be non-Archimedean normed spaces over $\mathbb{K}$ with $|2|<1$. If $Y$ is complete and for some $0 \leq r<1, f: X \rightarrow Y$ satisfies the condition

$$
\|D f(x, y)\| \leq \theta\left(\|x\|^{r}+\|y\|^{r}\right)
$$

for all $x, y \in X$. Then there exists a unique quadratic-additive mapping $T: X \rightarrow Y$ such that

$$
\|f(x)-T(x)\| \leq \begin{cases}|2|^{-1-r} \theta\|x\|^{r} & \text { if } 0<r<1, \\ 2|2|^{-1} \theta & \text { if } r=0 .\end{cases}
$$

Proof. Let $\varphi(x, y)=\theta\left(\|x\|^{r}+\|y\|^{r}\right)$. Since $|2|<1$ and $1-r>0$,

$$
\lim _{n \rightarrow \infty}|2|^{n} \varphi\left(2^{-n} x, 2^{-n} y\right)=\lim _{n \rightarrow \infty}|2|^{n(1-r)} \varphi(x, y)=0
$$

for all $x, y \in X$. Therefore $f$ and $\varphi$ satisfy the conditions of Theorem 2.3. It is easy to see that $\tilde{\varphi}(x)=|2|^{-1-r} \theta\|x\|^{r}$ if $0<r<1$ and $\tilde{\varphi}(x)=2|2|^{-1} \theta$ if $r=0$. By Theorem 2.3 there is a unique quadratic-additive mapping $T: X \rightarrow Y$ satisfying (2.14). 


\section{References}

[1] T. Aoki, On the stability of the linear transformation in Banach spaces, J. Math. Soc. Japan 2 (1950), 64-66.

[2] D. H. Hyers, On the stability of the linear functional equation, Proc. Natl. Acad. Sci. USA 27 (1941), 222-224.

[3] M.S. Moslehian and Th. M. Rassias, Stability of functional equations in nonArchimedean spaces, Appl. Anal. Discrete Math. 1 (2007), 325-334.

[4] Th. M. Rassias, On the stability of the linear mapping in Banach spaces, Proc. Amer. Math. Soc. 72 (1978), 297-300.

[5] S.M. Ulam, A Collection of Mathematical Problems, Interscience, New York, 1960.

Chang-Ju Lee

Department of Mathematics Education, Gongju National University of Education,

Gongju 314-060, Republic of Korea.

E-mail: chjlee@gjue.ac.kr

Yang-Hi Lee

Department of Mathematics Education, Gongju National University of Education,

Gongju 314-711, Republic of Korea.

E-mail: yanghi2@hanmail.net 